\title{
A New Statistical Restoration Method for Spatial Domain Images
}

\author{
Arijit Sur ${ }^{1, \star}$, Piyush Goel ${ }^{2}$, and Jayanta Mukherjee ${ }^{2}$ \\ 1 Department of Computer Science and Engineering, \\ Indian Institute of Technology, Guwahati-781039, India \\ 2 Department of Computer Science and Engineering, \\ Indian Institute of Technology, Kharagpur-721302, India \\ arijit@iitg.ernet.in, \{piyush,jay\}@cse.iitkgp.ernet.in
}

\begin{abstract}
In this papen, a new algorithm is proposed for restoring first order statistics of the cover image after steganographic embedding in spatial domain on gray scale images. The main motivation of this paper is to prevent first order statistics based steganalytic attack in spatial domain. We provide experimental results to show that the proposed scheme gives better performance than existing restoration methods especially for non-Gaussian cover distribution. A few state of art steganalytic attacks based on first order image statistics are considered and it is experimentally shown that proposed scheme has outperformed the existing schemes against those attacks.
\end{abstract}

\section{Introduction}

Statistical undetectability is one of the main aspects of a steganographic algorithm. In steganographic research several algorithms have been proposed for preserving statistical features of the cover for achieving more security. Recently Solanki et al. 1] have proposed a statistical restoration method where a portion of cover coefficients is allocated for embedding and another portion is used to restore the statistics. To ensure Minimum Mean Square Error (MMSE) criteria while modifying the histogram, all the bins of the target histogram are compensated in an increasing order by mapping the input data with values in increasing order [2. To the best of our understanding, histogram restoration in this fashion limits the application of the algorithm to only Gaussian cover. Further the algorithm shows some erratic behavior in low probability tail areas. The proposed scheme tries to overcome this limitation of a Gaussian cover assumption and provides better restoration of image histogram for non-Gaussian cover distribution as well. The rest of the paper is organized as follows: In section 2, we formalize the important definitions used in the restoration scheme, the detailed algorithm and experimental results are given in section 3, the application of the scheme towards the steganographic algorithms as well as a new steganographic scheme is proposed in section 4 . The paper is concluded in section 5 .

\footnotetext{
* Corresponding author.

${ }^{1}$ The first author gratefully acknowledge the support received from Infosys Technologies Ltd., Bangalore, under the Infosys Fellowship Award.
} 


\section{Mathematical Formulation of Proposed Scheme}

The proposed restoration scheme is dependent on the embedding scheme. The whole idea of embedding and restoring is that some of image pixels are used for embedding and rest are used for restoration.

Let the cover image, stego image (i.e. embedded but not yet compensated) and compensated stego image (stego image after compensation) be defined by C, S and R respectively. Suppose $C_{i j}, S_{i j}$ and $R_{i j}$ represent the $(i, j)^{t h}$ pixel of $\mathrm{C}, \mathrm{S}$ and $\mathrm{R}$ images respectively $(0<i<m, 0<j<n, \mathrm{~m}$ is number of rows and $\mathrm{n}$ is number of columns of image matrices). Histogram $(h(I))$ of an image $I$ can be represented as $h(I)=\{h(0), h(1), h(2), \ldots, h(L-1)\}$

Definition 1 (Embed Matrix $(\Psi))$. It is a $m \times n$ characteristic matrix representing whether a pixel has been used for embedding or not. $\Psi(i, j)=1$ if $(i, j)^{\text {th }}$ pixel is used for embedding and $\Psi(i, j)=0$ if $(i, j)^{\text {th }}$ pixel is not used for embedding.

Definition 2 (Compensation $\operatorname{Vector}(\Omega)$ ). It is a one dimensional vector with length $L$ where $L$ is number of existing gray levels in the cover image $(C) . \Omega(k)$ $=u$ means that $u$ number of pixels with gray value $k$ can be used for restoration.

Definition 3 (Changed Matrix $(\Gamma)$ ). It is a $L \times L$ matrix where $L$ is number of existing gray levels in the cover image $(C) . \Gamma(x, y)=\lambda$ means during embedding $\lambda$ number of pixels are changed from gray value $x$ to gray value $y$.

Changed Matrix $(\Gamma)$ is computed as given below:

$$
\Gamma(x, y)=\sum_{i=0}^{m} \sum_{j=0}^{n} e q\left(C_{i j}, x\right) \times e q\left(S_{i j}, y\right) \times \epsilon_{i j}
$$

where $e q(a, b)=1$ if $\mathrm{a}=\mathrm{b}$ and $e q(a, b)=0$ if $a \neq b$.

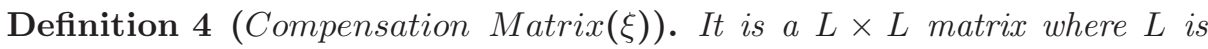
number of existing gray levels in the cover image $(C) . \xi(x, y)=\lambda$ means during embedding number of times $x$ is changed to $y$ minus number of times $y$ changes to $x$ is $\lambda$.

Compensation Matrix $(\xi)$ has been formed as following:

$$
\xi=U T\left(\Gamma-\Gamma^{T}\right)
$$

where $U T(M)$ means upper triangulation of matrix $M$.

\section{Proposed Restoration Scheme}

\subsection{Algorithm}

The detailed restoration algorithm is described below using the matrices and vector defined in the previous section. 


\section{Algorithm Restoration}

begin

for each element $\xi(i, j)$ of compensation matrix

do

\{

Step1: $\mathrm{k}=\xi(i, j)$

Step2: If $k>0, k$ number of pixels with gray value $i$ from the set of pixels used for compensation are changed to gray value $j$ for full compensation. If $k<0$, reverse operation is done i.e. $k$ number of pixels with gray value $j$ from the set of pixels used for compensation are changed to gray value $i$ for full compensation.

Step3: Modify the Compensation Vector $(\Omega)$ to reflect the pixel changs under taken in step 2 as in Eq.(3) below

$$
\Omega(i)= \begin{cases}\Omega(i)-k & \text { if } \Omega(i)>k \\ 0 & \text { if } \Omega(i) \leq k\end{cases}
$$

${ }^{*}$ Here we assume that for $\Omega(i)<k$, full compensation is not possible. Further research can be possible to improve this situation.*/

\}

\section{End Algorithm Restoration}

\subsection{Restoration with Minimum Distortion}

The additional noise added due to compensation is an important issue. The goal is to design a restoration procedure in such a way that additional noise should be kept minimum. In the proposed compensation procedure, the noise introduced depends on the embedding algorithm used. The total noise $(\eta)$ introduced at the time of restoration can be estimated by

$$
\eta=\sum_{i=0}^{L-1} \sum_{j=1}^{a b s[\hat{h}(i)-h(i)]} a b s\left(i-k_{j}\right)
$$

where $\hat{h}(i)$ and $h(i)$ is the histogram of the stego and cover images respectively. $L-1$ is the no. of bins in the histogram. $k_{j}\left(0 \leq k_{j} \leq L-1\right)$ is a bin that is used to repair atleast one unit of data in $i^{\text {th }}$ bin.

Lemma 1. With any restoration scheme the minimum total noise $\sum_{i=0}^{L-1}$ abs $[\hat{h}(i)-h(i)]$.

Proof. The total noise $(\eta)$ introduced at the time of restoration is

$$
\eta=\sum_{i=0}^{L-1} \sum_{j=1}^{a b s[\hat{h}(i)-h(i)]} a b s\left(i-k_{j}\right)
$$


where $1 \leq a b s\left(i-k_{j}\right) \leq L-1 . \eta$ is minimum when $a b s\left(i-k_{j}\right)=1$. Putting $\operatorname{abs}\left(i-k_{j}\right)=1$ in Eq. 5 we get

$$
\eta=\sum_{i=0}^{L-1} a b s[\hat{h}(i)-h(i)]
$$

Lemma 2. With proposed restoration scheme the total noise $(\eta)$ is minimum if maximum noise per pixel due to embedding is 1 .

Proof. Since the proposed restoration scheme is based on simple pixel swapping strategy i.e. if a the gray level value $\alpha$ of a pixel is changed to $\beta$ during steganographic embedding, at the time of restoration, a pixel with gray level value $\beta$ is changed to $\alpha$.

During embedding with \pm 1 embedding, the gray level value of a pixel, $x$ can be changed into either $x+1$ or $x-1$. Hence during restoration the proposed scheme restores bin $x$ value is repaired from either bin $x+1$ or $x-1$ according to embedding. It is to be noted that maximum noise that can be added during restoration for one member of a bin is atmost 1 since we are using only the neighboring bins for compensation. Hence, with \pm 1 embedding scheme (or any other steganographic scheme where noise added during embedding per pixel is atmost 1), the proposed scheme increments or decrements gray value by 1 i.e. $\operatorname{abs}\left(i-k_{i}\right)=1$.

From Eq. (6), the total noise $(\eta)$ introduced at the time of restoration is

$\eta=\sum_{i=0}^{L-1} \sum_{j=1}^{a b s[\hat{h}(i)-h(i)]} a b s\left(i-k_{j}\right)$

since in the proposed restoration scheme $a b s\left(i-k_{i}\right)=1$, putting this value in above equation, we get

$$
\begin{aligned}
& \eta=\sum_{i=0}^{L-1} \sum_{j=1}^{a b s[\hat{h}(i)-h(i)]}(1) \\
& =\sum_{i=0}^{L-1} a b s[\hat{h}(i)-h(i)]
\end{aligned}
$$

So from Lemma 1 and 2, we can conclude that with proposed restoration scheme minimum amount of noise is added during restoration if maximum noise per pixel due to embedding is atmost 1.

\section{Experimental Results}

\subsection{Comparison with Existing Method(s)}

In our experiments, LSB matching at embedding rate $0.125 b p p$ is used as the steganographic embedding method on two standard images as Dinosaur and Baboon. In Fig. 1 and 2, Histogram, Difference Histogram Before Compensation, Difference Histogram After Compensation by Solanki's Method and Difference Histogram After Compensation by Proposed Method are given for Dinosaur and Baboon images respectively. It may be seen from Figs. 1 and 2 that the proposed scheme provides better restoration than Solanki. et. al's scheme. 


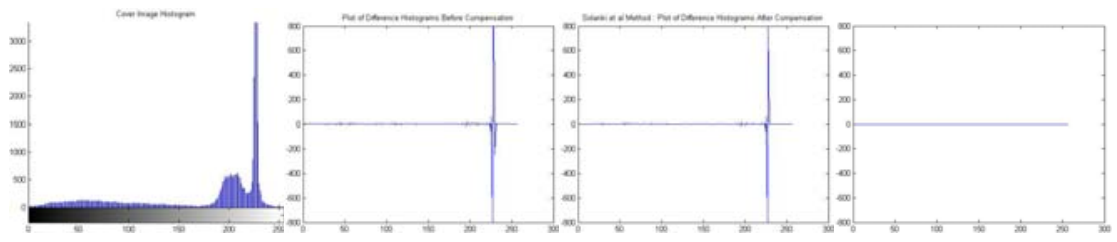

Fig. 1. Histogram, Difference Histogram Before Compensation, Difference Histogram After Compensation by Solanki's Method and Difference Histogram After Compensation by Proposed Method respectively for Dianosaur Image

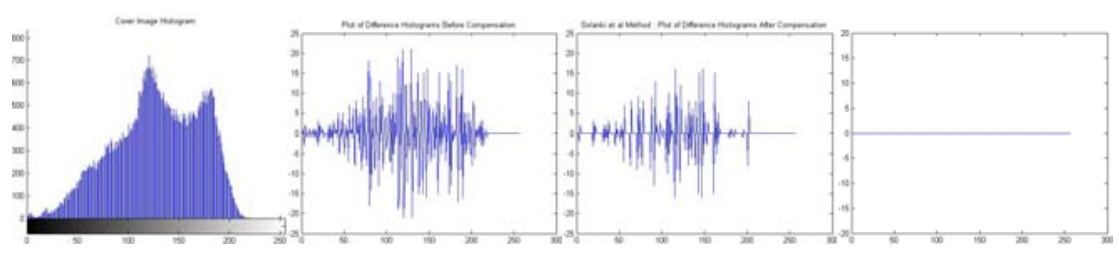

Fig. 2. Histogram, Difference Histogram Before Compensation, Difference Histogram After Compensation by Solanki's Method and Difference Histogram After Compensation by Proposed Method respectively for Baboon Image

\subsection{Application towards Steganography}

To analyze the applicability of the proposed scheme towards more secure steganographic algorithm, the LSB matching is used as steganographic embedding scheme on images images from UCID dataset [5]. Some of the state of art targeted attacks like Ker's calibrated HCF [3], HCF of Adjacency Histogram based attacks [3] and Jun Zhang et al 's high frequency noise based attack [4] are considered for experimentation. To evaluate the steganographic security using our proposed scheme, we have used Area under the Receiver Operating Characteristic Curve $\left(A_{R O C}\right)$ and the Detection accuracy $\left(P_{\text {detect }}\right)$ [6].

In Figs. 3 and 4 , we have shown the $P_{\text {detect }}$ and $\left(A_{R O C}\right)$ plots for comparing the proposed restoration scheme with the restoration scheme proposed in [1]. It
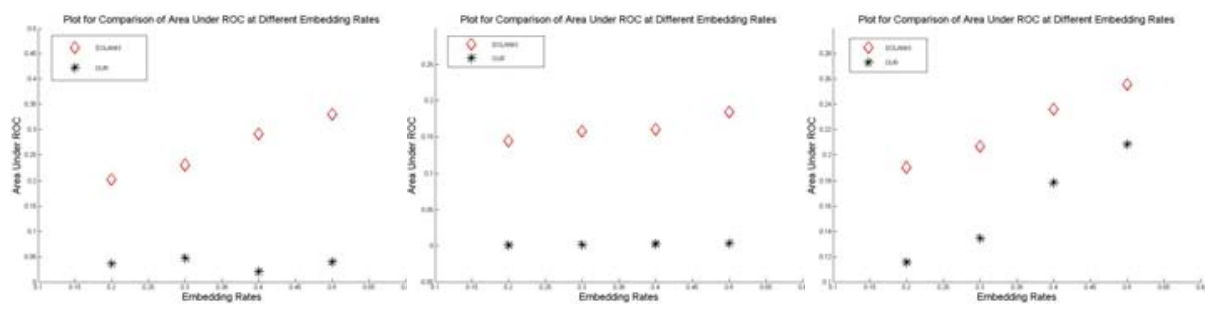

Fig. 3. Comparison of Area under ROC against Jun Zhang et al 's targeted attack, HCF Calibration Attack and HCF Adjacency Attack 

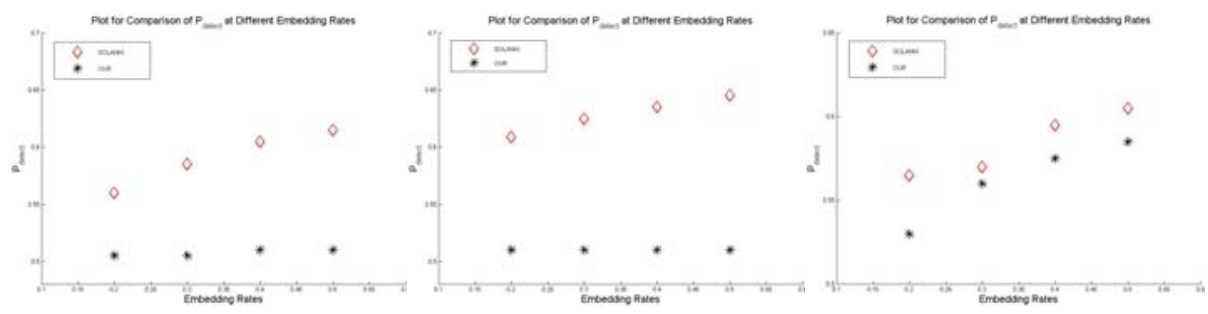

Fig. 4. Comparison of $P_{\text {detect }}$ against Jun Zhang et al 's targeted attack, HCF Calibration Attack and HCF Adjacency Attack

can be observed from Fig. 3 and 4, our proposed method geartly outperforms the competing scheme against mentioned targeted attacks.

\section{Conclusion}

In this paper we have proposed a new statistical restoration scheme which can be used for preserving the histogram i.e. first order statistics of the cover image after embedding and thus making the data hiding process robust against first order statistic based steganalytic attack. Moreover the proposed scheme does not assume any particular distribution for the cover image and hence gives better performance than scheme given in 1] especially for non-Gaussian covers. It must be mentioned that the additional noise added during restoration depends on the embedding algorithm for proposed scheme and is a topic of future research.

\section{References}

1. Solanki, K., Sullivan, K., Madhow, U., Manjunath, B.S., Chandrasekaran, B.S.: Statistical Restoration for Robust and Secure Steganography. In: Proc. IEEE International Conference on Image Processing, Genova, Italy (September 2005)

2. Tzschoppe, R., Bauml, R., Eggers, J.J.: Histogram modifications with minimum MSE distortion. Tech. rep., Telecom. Lab., Univ. of Erlangen-Nuremberg (December 2001)

3. Ker, A.: Steganalysis of LSB matching in grayscale images. IEEE Signal Process. Lett. 12(6), 441-444 (2005)

4. Zhang, J., Cox, I.J., Doerr, G.: Steganalysis for LSB Matching in Images with Highfrequency Noise. In: Proc. IEEE 9th Workshop on Multimedia Signal Processing, MMSP 2007, October 1-3, September 16-October 19, pp. 385-388 (2007)

5. Schaefer, G., Stich, M.: UCID - An Uncompressed Colour Image Database. In: Proc. SPIE, Storage and Retrieval Methods and Applications for Multimedia, vol. 5307, pp. $472-480$ (2004)

6. Solanki, K., Sarkar, A., Manjunath, B.S.: YASS: Yet Another Steganographic Scheme that Resists Blind Steganalysis. In: Furon, T., Cayre, F., Doërr, G., Bas, P. (eds.) IH 2007. LNCS, vol. 4567, pp. 16-31. Springer, Heidelberg (2008) 